\title{
Análise da informação sobre a leishmaniose visceral disponível em portais brasileiros da rede mundial de computadores - Internet
}

\author{
Analysis of the information on visceral leishmaniasis available \\ through Brazilian portals of the worldwide web - Internet
}

Camila Luciano Narciso de Souza', Zélia Profeta Luz ${ }^{1}$ e Ana Rabello ${ }^{1}$

\begin{abstract}
RESUMO
Com o objetivo de analisar a adequação da informação sobre leishmaniose visceral disponível em portais brasileiros, realizou-se através de portal de busca, levantamento, seleção e análise do conteúdo das páginas eletrônicas. Foram identificadas 24 páginas com objetivos informativos, sendo oito governamentais, 10 comerciais e seis classificadas como outros. Utilizando-se um formulário, foram avaliados os conteúdos relativos à transmissão, reservatório, controle e doença, contendo tópicos considerados essenciais. Os manuais normativos do Ministério da Saúde e a literatura científica foram definidos como padrões de informação. Observou-se, em todas as páginas, elevados percentuais de ausência de informações referentes aos tópicos discriminados em cada um dos quatro blocos de conteúdos. A análise de adequação das informações, quando estas estavam presentes, mostrou 100\% de adequação nas páginas governamentais e outros. Entretanto, nas páginas comerciais foram elevados os percentuais de informações incorretas ou incompletas, principalmente no bloco doença. De modo geral, o conteúdo sobre a leishmaniose visceral nas páginas eletrônicas analisadas foi considerado pouco informativo.
\end{abstract}

Palavras-chaves: Leishmaniose visceral. Internet. Informação. Portais brasileiros.

\begin{abstract}
With the aim of analyzing the adequacy of the information on visceral leishmaniasis that is available through Brazilian portals, a survey was conducted through a search portal, with selection and analysis of electronic page content. Twenty-four pages with informative purposes were identified, of which eight belonged to government bodies, 10 were commercial and six were classified as others. Using a systematized analysis form, the content of topics considered essential, relating to transmission, reservoirs, control and disease, was evaluated. The regulatory manuals of the Ministry of Health and the scientific literature were defined as the standards for information. High percentages of absences of information relating to the topics discriminated in each of the four content categories were observed in all of the pages. Analysis of the adequacy of the information, when it was present, showed $100 \%$ adequacy on the pages of the government bodies and the others. However, the commercial pages displayed high percentages of incorrect or incomplete information, particularly in the disease category. Overall, the content on leishmaniasis on the electronic pages analyzed was considered to be of low informative value.
\end{abstract}

Key-words: Visceral leishmaniasis. Internet. Information. Brazilian portals.

Os avanços das tecnologias da informação vêm causando mudanças na forma de comunicação em quase todo o mundo, embora com intensidade diferenciada em função do nível de desenvolvimento das sociedades e de fatores políticos, sociais e culturais.

A internet, um dos mais relevantes entre estes avanços tecnológicos, teve seu início no final da década de sessenta e alcançou notável progresso com a criação da World Wide Web em 1992. A rede de computadores é utilizada para inúmeras atividades e consiste em fonte de informação democrática para a maioria dos grupos populacionais que a ela têm acesso. A partir da digitação de duas ou três palavras nos serviços de busca é possível encontrar múltiplas respostas para qualquer tema.

A internet teve sua origem nos Estados Unidos em 1969. No Brasil, como em outros países do mundo, a rede inicialmente interligava laboratórios de pesquisa e estava vinculada à área acadêmica e militar, expandindo-se comercialmente a partir de $1994^{1113}$. 0 número de usuários de Internet no mundo em junho

\footnotetext{
1. Laboratório de Pesquisas Clínicas, Centro de Referência em Leishmanioses, Centro de Pesquisas René Rachou, Fundação Oswaldo Cruz, Belo Horizonte, MG. Apoio financeiro: CNPq, FAPEMIG, FIOCRUZ

Endereço para correspondência: Dra. Ana Rabello. Laboratório de Pesquisas Clínicas/CPqRR/FIOCRUZ. Av Augusto de Lima 1715 Barro Preto, $30190-002$ Belo Horizonte, MG. Tel: 5531 3349-7782; Fax: 3295-3115

e-mail: ana@cpqrr.fiocruz.br

Recebido para publicação m 05/12/2007

Aceito em 26/07/2008
} 
de 2006 era de cerca de um bilhão e destes, 26 milhões eram do Brasil, representando cerca de $15 \%$ da população brasileira (http://www.internetworldstabs.com).

De acordo com a Pesquisa Nacional por Amostras de Domicílios (PNAD), em 2005, a utilização de Internet no Brasil concentrou-se no grupo etário mais jovem, de 10 a 17 anos. Além disso, o nível de instrução dos usuários foi acentuadamente mais elevado quando comparado ao das pessoas que não usavam a rede. Observou-se ainda que dentre os usuários com mais de 10 anos de idade, $72 \%$ acessou a internet com finalidade de educação e aprendizado (http://www.ibge.gov.br).

A internet é principalmente utilizada pela população urbana e pode auxiliar na disseminação de informação de diferentes áreas do conhecimento, incluindo as relativas à saúde, prevenção e controle de doenças. Estima-se que exista informação sobre saúde em 20.000 a 100.000 web sites relacionados ${ }^{6}$. Além disso, cerca de 30-50\% dos usuários procuram regularmente informações sobre saúde ${ }^{14}$.

A web está se tornando uma fonte de informação importante, não só para pacientes, mas para seus familiares, na tentativa de obtenção de informações válidas sobre as causas, tratamento e controle de doenças ${ }^{2}$. Entretanto, como não há procedimento de validação ou de garantia de qualidade da informação disponibilizada, o conteúdo incorreto pode comprometer a promoção adequada de práticas que contribuam para a redução de situações associadas ao adoecimento ${ }^{3}$.

A leishmaniose visceral (IV) é uma doença grave que, se não diagnosticada e tratada, éfatal em $100 \%$ dos casos ${ }^{5}$. No Brasil, a doença que era predominantemente rural nos anos 80, a partir dos anos 90 se caracterizou como doença urbana. São notificados no país, 3.187 casos/ ano, com incidência anual média de 2/100.000 habitantes. Embora 0 tratamento da LV seja eficaz na maioria dos casos, a letalidade da doença no Brasil é elevada, com variação de 5,8\% a 7,4\% nos últimos cinco anos (2001-2005) ${ }^{9}$. As medidas de prevenção e controle se baseiam essencialmente em manejo ambiental, tratamento dos casos humanos, eliminação do cão com sorologia reagente, principal reservatório em áreas urbanas e combate ao vetor.

0 repasse da informação correta sobre $\mathrm{a} \mathrm{LV}$ à população por diferentes meios de comunicação pode constituir ferramenta importante para as ações de prevenção e controle da doença ${ }^{8}$.

No presente trabalho, objetivou-se analisar a qualidade da informação sobre a LV disponibilizada em portais de internet brasileiros.

\section{MATERIAL E MÉTODOS}

Realizou-se o levantamento de portais que apresentam informações sobre a leishmaniose visceral, através do portal de busca Google (www.google.com.br) no período de março a outubro de 2005.

A palavra-chave utilizada foi leishmaniose visceral. Em seguida foi feita a seleção de portais com objetivos informativos. Foram analisados conteúdo e qualidade da informação. Tomou-se como padrão de informação adequada, aquelas contidas nos Manuais Normativos do Ministério da Saúde e a literatura científica.
Para o estudo de conteúdo, usou-se como instrumento um formulário (Tabela 1), com 27 tópicos, agrupados em quatro blocos: a) transmissão, b) reservatório, c) controle e d) doença. As informações foram avaliadas por duas das autoras. Em caso de julgamentos discordantes, a página foi revisitada e o tópico discutido até o consenso. A informação relativa a cada tópico foi classificada como ausente, correta ou incorreta/incompleta.

Tabela 1 - Formulário usado para avaliação das informações no portais de internet brasileiros.

\section{1) Transmissão}

T1: A leishmaniose visceral é causada por um microorganismo chamado Leishmania

T2: A transmissão da leishmaniose ocorre através de picada de inseto

T3: 0 inseto transmissor é pequeno, claro e apresenta pelos brancos nas asas

T4: 0 inseto transmissor vive em ambiente escuro e úmido

T5: 0 acúmulo de material orgânico (lixo) favorece a proliferação do inseto transmissor

T6: 0 inseto vetor tem hábito alimentar vespertino (pica as pessoas principalmente ao final da tarde)

T7: Galinheiros e outros criadouros de aves atraem os insetos vetores para 0 domicílio

\section{2) Reservatório}

R1: Reservatórios são animais que estão infectados pela Leishmania e atuam como fonte de infecção para os insetos vetores

R2: 0 cão é o principal reservatório em regiões urbanas

R3: Roedores e marsupiais são os principais reservatórios silvestres

R4: Os reservatórios são infectados pela picada de insetos vetores

R5: É possível saber se um cão está infectado através de exame de sangue

R6: Um cão infectado e transmitindo a doença pode aparentar-se saudável

R7: Os principais sintomas da leishmaniose canina são emagrecimento, perda de pelos e/ou lesões de pele

R8: Apesar de ser realizado por alguns veterinários, o tratamento canino não é recomendado pelo Ministério da Saúde

R9: Apesar de ser realizada por algumas clínicas veterinárias, a vacinação canina não é recomendada pelo Ministério da Saúde

\section{3) Controle}

C1: A borrifação do domicílio com inseticidas é realizada pela Secretaria Municipal de Saúde quando há um caso de doença notificado

C2: 0 cão com exame de sangue positivo deve ser submetido a eutanásia pela Secretaria Municipal de Saúde

C3: A limpeza da área domiciliar, com retirada de dejetos orgânicos é uma das medidas de controle da doença

\footnotetext{
4) Doença

D1: Os principais sintomas de leishmaniose visceral são febre, fraqueza, emagrecimento e palidez

D2: Ao exame médico, o fígado e o baço poderão estar aumentados

D3: 0 exame de sangue (hemograma) pode indicar anemia (baixa de hemácias ou glóbulos vermelhos), baixa de leucócitos (glóbulos brancos) e baixa de plaquetas

D4: Para confirmar a suspeita da doença são necessários exames de laboratório: exame de sangue ou pesquisa do parasita em material de orgãos (medula óssea ou baço)

D5: A leishmaniose visceral tem tratamento e na maioria das vezes, o paciente fica curado

D6: Quanto mais rápido for feito o diagnóstico da doença, maior será a chance de cura

D7: 0 medicamento é fornecido pelo governo

D8: A doença não pega de pessoa para pessoa

D9: 0 serviço de saúde pública precisa ser notificado a cada caso de leishmaniose para as providências devidas
} 
Tabela 2 - Relação dos portais com informação sobre a leishmaniose visceral, acessados no período de 17 de março a 18 de outubro de 2005.

\begin{tabular}{|c|c|}
\hline Identificação do portal & Endereço \\
\hline \multicolumn{2}{|l|}{ Portais governamentais } \\
\hline Centro de Vigilância Epidemiológica - SES-SP & www.cve.saude.sp.gov.br/doc_tex/20. pdf \\
\hline SUCEN - Superintendência de Controle de Endemias & www.sucen.sp.gov.br/doenças/leish_visc/texto_leishmaniose_visceral_americana.htm \\
\hline \multicolumn{2}{|l|}{ Estado de São Paulo } \\
\hline SESPA - Secretaria Executiva de Saúde Pública do Pará & www.sespa.pa.gov.br/educação/vetores.htm \\
\hline Manual Técnico do Instituto Pasteur & www.pasteur.saude.sp.gov.br/extras/manual_05.pqf \\
\hline Secretaria de Saúde do Estado do Mato Grosso do Sul & www.saude.ms.gov.br/Saude/outros/leishmanioseVisceralAmericana.htm \\
\hline Secretaria de Saúde do Estado do Rio de Janeiro & www.saude.rj.gov.br/Açoes/Leish.shtml \\
\hline Secretaria de Saúde do Rio de Janeiro & www.saude.rj.gov.br/Guia_sus_cidadao/pg_51,shtml \\
\hline Centro de Vigilância Epidemiológica - SUCEN - SP & www.sucen.sp.gov.br/docs_tec/programalva20 \\
\hline \multicolumn{2}{|l|}{ Portais comerciais } \\
\hline Empresa Comercial De Cicco S/C LTDA & www.saudeanimal.com.br/artigo99.htm \\
\hline Empresa Dog Times & www.dogtimes.com.br/leishmaniose.htm \\
\hline Nós e as radiações \& Patologia & www.nuclear.radiologia.com.br/trabalho/estudo/patology/patogeno/leishman.htm \\
\hline Instituto Brasileiro de Produção Sustentável e Direito Ambiental & www.ibps.com.br/index.asp?idnoticia $=1813$ \\
\hline Saúde em Movimento & www.saudeemmovimento.com.br/conteúdos/conteúdo_frame.asp?cod_noticia=1006 \\
\hline UOL-Folha Online - Folha de São Paulo & www1.uol.com.br/fol/geral/ult13012000234.htm \\
\hline Portal Saúde animal & www.saudeanimal.com.br/artigo99a.htm \\
\hline Portal Ambiente Brasil & www.ambientebrasil.com.br/.../urbano/index.html\&conteudo=./urbano/artigos/leishmaniose.htm \\
\hline Site Oficial do Dr. Drauzio Varella & www.drauziovarella.com.br/entrevistas/dtropicais6.asp \\
\hline Empresa Shering Plough Coopers & www.especialpet.com.br/Script/CepCaesLeishmaniose.asp \\
\hline \multicolumn{2}{|l|}{ Portais outros } \\
\hline The University of Georgia- College of Veterinary & www.vet.uga.edu/vpp/NSEP/Brazil2002/leishmaniose/Port \\
\hline \multicolumn{2}{|l|}{ Medicine, convênio com Universidade Federal de Minas Gerais } \\
\hline Grupo Whippets & whippetp.no.sapo.pt/leishmaniose.htm \\
\hline FAMERP - Faculdade de Medicina de São José do Rio Preto & www.informedgrp7.famerp.br/default.asp?id $=1 \& \mathrm{ACT}=5$ content $=3 \& \mathrm{mnu}=1$ \\
\hline UFV-Universidade Federal de Viçosa & www.insecta.ufv.br/Entomologia/ent/disciplina/ban \%... \\
\hline Ministério da Saúde-Fundação Oswaldo Cruz & www.bibmanquinhos.cict.fiocruz.br/pseriedoencas.htm \\
\hline UNESCO-Joinville SC & www.mundofisico.joinville.udesc.br/Enciclopedia/16 \\
\hline
\end{tabular}

\section{RESULTADOS}

A partir da palavra-chave leishmaniose visceral foram encontradas 257 citações e endereços eletrônicos, que foram visitados. Desses, 24 páginas tinham objetivos informativos, sendo oito páginas governamentais, dez comerciais e seis classificadas como outros, por não se enquadrarem nas duas anteriores (Tabela 2).

De modo geral, observou-se elevada freqüência de informações ausentes em relação a todos os tópicos pesquisados, em todas as páginas analisadas. As Tabelas 2 e 3 resumem estes resultados. Observa-se que nas páginas governamentais o percentual médio de informações ausentes variou de 54\% a $69 \%$. Nas páginas comerciais e na classificada como outros os valores variaram de $60 \%$ a $78 \%$.

A análise dos diferentes tópicos em cada um dos quatro blocos investigados mostrou que a ausência de informações no bloco relacionado à transmissão foi variável e elevada no conjunto de páginas pesquisadas (Figura 1A). Os menores percentuais de informações ausentes nos três portais (gov, com e outros) foram observados nos tópicos T1 e T2 que abordavam, respectivamente, o agente etiológico e 0 aspecto do inseto
Tabela 3 - Informações sobre leishmaniose visceral nos portais brasileiros. Percentual de informações ausentes nos blocos de conteúdos transmissão, reservatório, controle e doença.

\begin{tabular}{lllr}
\hline Bloco & \multicolumn{3}{c}{$\begin{array}{c}\text { Percentual médio e valores máximos e mínimos de informações } \\
\text { ausentes nas páginas \% médio (min-max) }\end{array}$} \\
\cline { 2 - 4 } & governamentais & comerciais & outros \\
\hline Transmissão & $57(25-100)$ & $60(0-100)$ & $60(0-100)$ \\
Reservatório & $69(38-88)$ & $63(30-100)$ & $63(33-83)$ \\
Controle & $54(50-63)$ & $63(40-90)$ & $78(67-100)$ \\
Doença & $56(13-88)$ & $73(50-100)$ & $78(50-100)$ \\
\hline
\end{tabular}

transmissor. Verificou-se menor (50\%) percentual de ausência de informações nas páginas governamentais quando comparadas com as demais ( $80 \%$ a $100 \%$ ) nos tópicos que abordavam as condições ambientais favoráveis para proliferação do vetor. Em nenhuma das páginas observou-se informação sobre associação do inseto vetor e presença de galinheiros e outros criadouros de aves (Figura 1A).

A Figura 1B mostra os resultados do bloco reservatório. De forma semelhante ao observado no bloco transmissão, verificou-se elevado percentual de informação ausente em todas as páginas. A informação que esteve mais presente, apenas nas páginas comerciais, foi a que abordava a forma de transmissão para 0 
cão (R4). Variou de 30\% nas páginas comerciais, 33\% em outros e 38\% nas páginas governamentais, a informação que abordava o principal reservatório em áreas urbanas (R2). Nos demais itens a ausência de informações variou de 50\% a mais de $80 \%$.

No bloco controle (Figura 1C), os menores percentuais de informações ausentes, nos três conjuntos de páginas, estavam relacionados ao sacrifício do cão. 0 pior desempenho foi observado nas páginas comerciais e em outros no tópico que abordava as medidas de combate químico ao vetor.

Em relação à doença (Figura 1D) os percentuais de informações ausentes foram bastante elevados, com exceção das páginas governamentais, em relação aos tópicos que tratavam respectivamente dos principais sinais e sintomas da $\mathrm{LV}(\mathrm{D} 1, \mathrm{D} 2)$ e da importância da notificação da doença pelos profissionais de saúde (D9). Nos outros itens, a análise global dos três conjuntos de páginas mostrou variação de ausência de informações de $50 \%$ a $100 \%$.
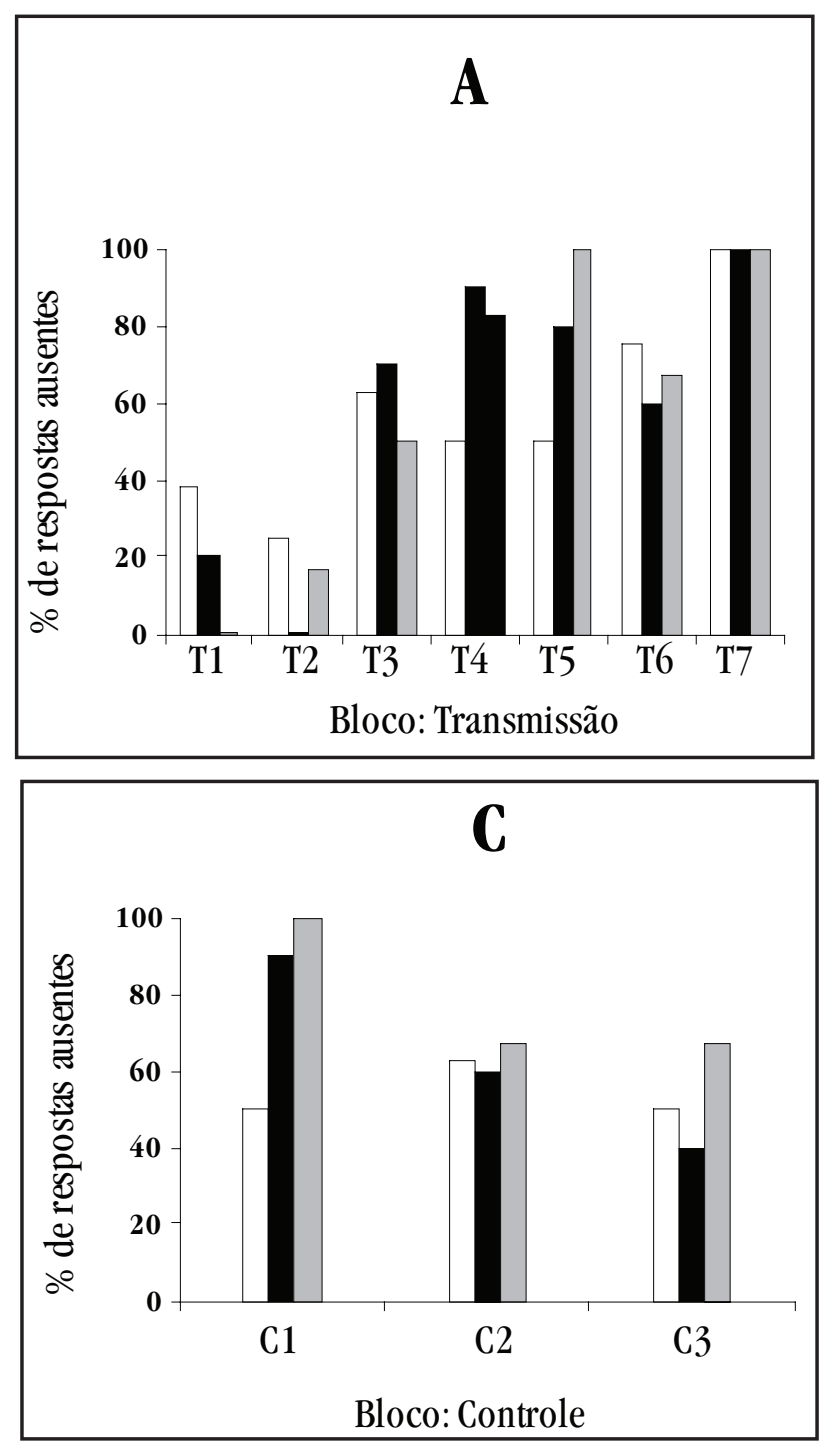

Legenda:
A adequação das informações, quando estas estavam presentes, foi analisada para cada tópico, sendo observados percentuais de $100 \%$ de informações corretas para todos os tópicos nas páginas governamentais e nas classificadas como outros. Nas páginas comerciais, $100 \%$ de informações corretas foram observadas para os blocos transmissão, reservatório e controle. Entretanto, no bloco que abordou a doença, verificou-se percentuais elevados de informações incorretas ou incompletas, que variaram de $50 \%$ a $60 \%$. Os tópicos nos quais foram observadas informações incorreta/incompletas foram D1, D4, D5 e D8, que tratavam respectivamente dos principais sintomas da doença, a necessidade de exame de diagnóstico laboratorial confirmatório, tratamento e transmissão homem a homem.

Informações extremamente equivocadas, como a exemplificada a seguir, não foram freqüentes. Estas preocupam ainda pela possibilidade de influenciar as práticas dos usuários: "Pessoas infectadas com o parasita, devem ser
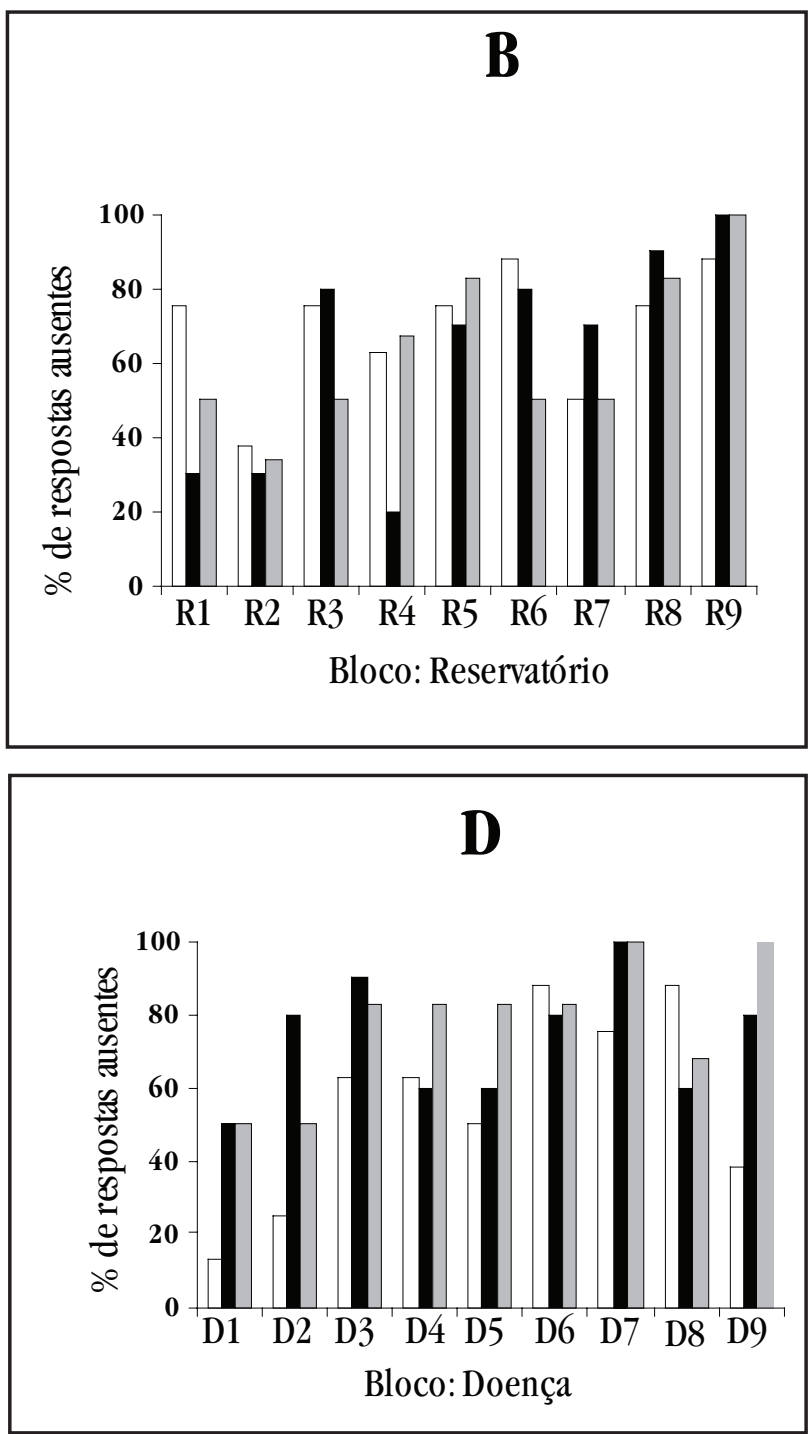

.$c 0 m$ outros

Figura 1 - Percentual de informações ausentes sobre a leishmaniose visceral nos blocos: transmissão (A), reservatório (B), controle (C) e doença (D) em páginas de portais de internet brasileiros. 
obrigatoriamente isoladas em local apropriado, a fim de ser impedido serem sugadas pelos vetores Flebótomos, interrompendo a cadeia evolutiva do protozoário. Trata-se, no entretanto, de uma doença chamada exótica, felizmente presente em pouquíssimos locais do território Brasileiro. Por essas características, quando diagnosticado um foco da doença, fazem-se necessárias medidas enérgicas para sua erradicação, sob pena de sua disseminação." http://www. saudeanimal.com.br/artig099.htm - acessado em junho de 2005 e em novembro de 2007.

\section{DISCUSSÃo}

Desde o seu surgimento até os dias atuais, a internet vem revolucionando o modo de transmitir informações em diferentes áreas do conhecimento ${ }^{11}$.

$\mathrm{Na}$ saúde, tem aumentado o uso da internet por pacientes, bem como seus familiares, para diferentes fins, seja de consulta, esclarecimento ou outros. Estudo envolvendo pacientes com distúrbios intestinais nos Estados Unidos mostrou que 50\% deles tinham acesso à internet e desses, 51\% utilizavam a rede para buscar informações relacionadas à sua doença ${ }^{2}$. Resultados semelhantes foram observados em um levantamento que envolveu 512 indivíduos que utilizavam medicina privada, e dos quais $274(53,5 \%)$ relataram ter usado a internet para informações médicas ${ }^{6} .0$ potencial da Rede Mundial de Computadores para a disseminação de informação e conseqüente prática mais saudável é amplo. Entretanto, na área de saúde, a adequação e a qualidade das informações veiculadas pela internet tem sido objeto de estudo de diferentes autores que observam ser a qualidade dessa informação variável ${ }^{6}{ }^{10}$. Análise da informação sobre rinite alérgica em 173 web-sites brasileiros mostrou que em 42 (24\%) as informações sobre a doença eram imprecisas, 102 (59\%) apresentaram informação incorreta/incompleta e 128 (74\%) não apresentaram a origem da informação de forma clara ${ }^{12}$.

De modo geral, no presente trabalho, foi baixo o desempenho das páginas nos portais analisados quanto à relevância da informação sobre a LV. Aspectos importantes incluídos nos tópicos selecionados para a análise da informação sobre transmissão, reservatório, controle e doença, necessários para a informação de qualidade e que pudesse contribuir para o entendimento da doença e sua complexidade não foram contemplados. Foi elevada a ausência de informações para os tópicos mencionados, em todas as páginas. Resultados semelhantes foram observados em um estudo sobre escoliose, que analisou 50 web-sites classificados como acadêmicos (44\%), comerciais (16\%), dirigidos para médicos (18\%), dirigidos para não médicos (10\%) e sem classificação (12\%) e concluiu que independentemente do tipo de portal, as informações sobre a escoliose foram limitadas e de pouco valor informativo ${ }^{9}$. A qualidade da informação sobre artrite reumatóide, disponibilizada em 69 portais da Internet também foi considerada variável, com o melhor desempenho para os portais com sufixo gov e .edu quando comparados aos portais .com e .org, entre outros ${ }^{1}$.
A frequiência de informação correta sobre a LV foi elevada nas páginas governamentais e em outros. Entretanto, chamou a atenção o elevado percentual de informações incorreta/ incompletas no bloco doença nas páginas comerciais. Este resultado é preocupante, pois já existem relatos na literatura científica sugerindo a associação da informação imprecisa e ou incorreta veiculada na Internet com danos à saúde. É importante destacar que a maioria dos portais outros eram portais de instituições de educação. Crocco e cols ${ }^{3}$ numa análise de revisão da literatura científica através de diferentes bases de dados, identificaram estudos que sugerem a associação de distúrbios emocionais e físicos com a informação obtida na Rede ${ }^{3}$. Os autores referidos mostraram ainda, em um relato de caso, a piora de quadro de diarréia em criança de um ano de idade que foi associada à informação obtida na Internet e que influenciou a conduta dos pais ${ }^{4}$.

Devido às características da LV no Brasil, com expansão geográfica, aumento do número de casos e letalidade elevada, a informação correta pode ser instrumento importante para as ações de prevenção e controle da doença. Mas, torna-se fundamental que a informação veiculada por qualquer meio de comunicação, em especial a internet devido à sua crescente utilização pela população, seja correta e de qualidade, precisa e baseada em evidências científicas.

No Brasil, não há legislação específica para regular o uso da Internet e dessa forma, a informação veiculada não é padronizada nem submetida a critérios de qualidade ${ }^{12}$.

De modo geral, as informações sobre a LV contidas nas páginas analisadas foram consideradas pouco relevantes e pouco informativas. Os resultados observados apontam para a importância da criação de mecanismos de disseminação de informação correta sobre a LV pela internet, aproveitando esta ferramenta útil para a prevenção e o controle da LV no país.

\section{AGRADECIMENTOS}

Camila Narciso, aluna do ensino médio do Colégio Instituto Municipal de Administração e Ciências Contábeis (IMACO) de Belo Horizonte, desenvolveu o trabalho durante o Programa de Vocação Científica (PROVOC) do Centro de Pesquisas René Rachou, Fundação Oswaldo Cruz (FIOCRUZ). Os autores agradecem aos estudantes de iniciação científica João Paulo Peixoto Pena Barbosa e Mariana Kolos Galuppo pela leitura do manuscrito e relevantes sugestões.

\section{REFERÊNCIAS}

1. Ansani NT, Vogt M, Henderson BAF, Mckaveney TP, Weber RJ, Smith RB, Burda M, Kwoh CK, Osial TA, Starz T. Quality of arthritis information on the Internet. American Journal Health Syss Pharm 62:1184-1189, 2005.

2. Bernard A, Morgan L, Stephanie H, Caren R, Desmond L, Sander V. A Systematic Review of patient inflammatory bowel disease information resources on the world wide web. American Journal of Gastroenterology 102 :1-8, 2007.

3. Crocco AG, Villasis-Keever M, Jadad AR. Analysis of cases of harm associated with use of health information on the Internet. JAMA 287:2869-2871, 2002. 
4. Crocco AG, Villasis-Keever M, Jadad AR. Two wrongs don't make a right: Harm aggravated by inaccurate information on the Internet. Pediatrics 109:522-523, 2002.

5. Desjeux P. Urbanization: an increasing risk factor for leishmaniasis. World Health Organization- Weekly Epidemiological Record 44:365-372, 2002.

6. Diaz JA, Griffith RA, Ng JJ, Reinert SE, Friedmann PD, Moulton AW. Patients' Use of the Internet for Medical Information. Journal of General Internal Medicine 17:180-185, 2002.

7. Fundação Nacional de Saúde. Guia de vigilância epidemiológica. Ministério da Saúde, Brasília, 527-539, 2002.

8. Luz ZMP, Schall V, Rabello A. Evaluation of a pamphlet on visceral leishmaniasis as a tool for providing diasease information to healthcare professionals and laypersons. Caderno de Saúde Pública 21:606-621, 2005.

9. Mathur S, Shanti N, Brkaric M, Sood V, Kubeck J, Paulino C, Merola AA.. Surfing for scoliosis: the quality of information available on the internet. Spine 30:26952700,2005
10. Ministério da Saúde. Manual de vigilância e controle da leishmaniose visceral. Secretaria de Vigilância em Saúde. Departamento de Vigilância Epidemiológica. Série A. Normas e Manuais Técnicos. Brasília, 2003.

11. Silva FB, Cassiani SHB, Zem-Macarenhas SH. A Internet e a enfermagem: Construção de um site sobre administração de medicamentos. Revista latino americana de enfermagem- Ribeirão Preto 9: 116-122, 2001.

12. Silva LVER, Mello-Júnior JF, Mion 0. Evaluation of Brazilian web site information on allergic rhinitis. Brazilian Journal of Othorhinolaryngology 71: 590-597, 2005 .

13. Sorj B. brasil@povo.com: a luta contra a desigualdade na sociedade da informação. Rio de Janeiro: Jorge Zahar Editora, Brasília, DF, UNESCO, 2003.

14. Thakurdesai PA, Kole PL, ParreK RP. Evaluation of the quality and contents of diabetes mellitus patient education on Internet. Patient Education and Counseling 52:309-313, 2004. 